\title{
Erratum
}

\section{A Concise Synthesis of (S)-2-(Fluorodiphenylmethyl)pyrrolidine: A Novel Organocatalyst for the Stereoselective Epoxidation of $\alpha, \beta$-Unsaturated Aldehydes}

Christof Sparr, Eva-Maria Tanzer, Julia Bachmann, Ryan Gilmour* Synthesis 2010, 1394.

The advance online publication (e-First) version of this article contained the incorrect stereochemistry for the epoxide product in the graphical abstract.

This mistake has been corrected for both the current online version and the print publication. 